\title{
The long non-coding RNA expression profile of Coxsackievirus A16 infected RD cells identified by RNA-seq
}

\author{
Yingying Shi ${ }^{1,2}$, Huilin Tu${ }^{1}$, Xiong Chen ${ }^{1}$, Yingying Zhang ${ }^{1}$, Liujun Chen ${ }^{1}$, Zhongchun Liư ${ }^{4}$ \\ Jiqun Sheng ${ }^{5}$, Song Han ${ }^{1}$, Jun Yin ${ }^{1}$, Biwen Peng ${ }^{1,3}$, Xiaohua He ${ }^{1,3}$, Wanhong Liu ${ }^{1,2} \bowtie$ \\ 1. Pathogenic Organism and Infectious Diseases Research Institute, School of Basic Medical Sciences, \\ Wuhan University, Wuhan 430071, China \\ 2. Hubei Province Key Laboratory of Allergy and Immunology, Wuhan 430071, China \\ 3. Hubei Provincial Key Laboratory of Developmentally Originated Disease, School of Basic Medical \\ Sciences, Wuhan University, Wuhan 430071, China \\ 4. Institute of Neuropsychiatry, Renmin Hospital, Wuhan University, Wuhan 430060, China \\ 5. College of Life Science and Technology, Hubei Engineering University, Xiaogan 432000, China
}

Coxsackievirus A16 (CVA16) is one of major pathogens of hand, foot and mouth disease (HFMD) in children. Long non-coding RNAs (IncRNAs) have been implicated in various biological processes, but they have not been associated with CVA16 infection. In this study, we comprehensively characterized the landscape of IncRNAs of normal and CVA16 infected rhabdomyosarcoma (RD) cells using RNA-Seq to investigate the functional relevance of IncRNAs. We showed that a total of 760 IncRNAs were upregulated and 1210 IncRNAs were downregulated. Out of these dysregulated IncRNAs, $43.64 \%$ were intergenic, $22.31 \%$ were sense, $15.89 \%$ were intronic, $8.67 \%$ were bidirectional, $5.59 \%$ were antisense, $3.85 \%$ were sRNA host IncRNAs and $\mathbf{0 . 0 5 \%}$ were enhancer. Six dysregulated IncRNAs were validated by quantitative PCR assays and the secondary structures of these IncRNAs were projected. Moreover, we conducted a bioinformatics analysis of an IncRNAs (ENST00000602478) to elucidate the diversity of modification and functions of IncRNAs. In summary, the current study compared the dysregulated IncRNAs profile upon CVA16 challenge and illustrated the intricate relationship between coding and IncRNAs transcripts. These results may not only provide a complete picture of transcription in CVA16 infected cells but also provide novel molecular targets for treatments of HFMD.

\section{KEYWORDS Coxsackievirus A16 (CVA16); RNA-Seq; Long non-coding RNA(IncRNA); gene expression}

\section{INTRODUCTION}

Coxsackievirus A16 (CVA16) is a positive single stranded RNA virus that belongs to the Picornaviridae family (Mao et al., 2014). It is one of the major causes of

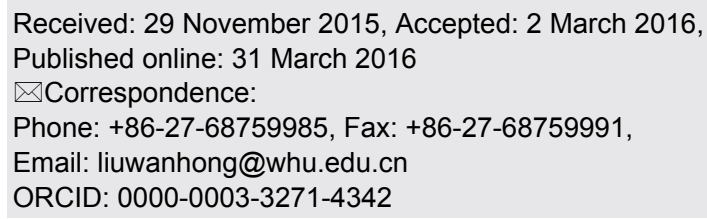

hand, foot, and mouth disease (HFMD) (Wei et al., 2014). HFMD is characterized by herpetic lesions on the hands, feet and oral mucosa of children under 5 years old (Mao et al., 2014). It is a serious public health threat to children in Asian-Pacific countries and leads to hundreds of deaths (Chen et al., 2013; Sun et al., 2014). Currently, the treatment and control of HFMD are only symptomatic. There are no effective medications or prophylactic vaccine (Mao et al., 2014). Although an extensive investigation has been performed and there has been progress with vaccines for EV71, no valid CVA16 vaccine is currently available (Ren et al., 2015). Increas- 
ing numbers of researches have been done to explore the possible pathogenic mechanisms of CVA16. However, the complete pathogenic mechanisms still remains largely unknown (Zhu et al., 2013; Shi et al., 2015).

Long non-coding RNAs (lncRNAs) are transcripts longer than 200 nucleotides without functional proteincoding capacity (Mattick and Makunin, 2006; Djebali et al., 2012; Fatica and Bozzoni, 2014). The majority of non-coding RNAs are IncRNAs (Djebali et al., 2012; Fatica and Bozzoni, 2014). IncRNAs can be divided into several categories based on their genomic region of origin and relative position to the protein-coding genes in the genome. These categories are as follows: sense, antisense, intronic, intergenic, bidirectional and enhancer (Mercer et al., 2009). IncRNAs are involved in various biological processes by functioning as a cis-tether, in cistargeting and trans-targeting, as an enhancer, a decoy, a scaffold, an allosteric modification, a co-activator or a co-repressor to modulate gene expression (Lee, 2012). Large-scale studies reported that these RNAs are widely transcribed from the genomes of most complex organisms (Mattick, 2011). It is estimated that approximately $90 \%$ of the mammalian genome is transcribed (Bertone et al., 2004; Djebali et al., 2012; Fatica and Bozzoni, 2014). However, messenger RNAs (mRNAs) and microRNAs (miRNAs), which are targeted in previous transcriptional profiling studies, account for approximately $1 \%$ of all transcribed species (Kapranov et al., 2007; Djebali et al., 2012). A large percentage of the mammalian genome is transcribed as non-coding RNAs, particularly IncRNAs (Mercer et al., 2009). The discovery of multiple classes of non-coding RNAs, that are pervasively transcribed in mammalian cells and involved in specific biological processes, challenges the traditional view of RNA as an intermediate between gene and protein (Wang and Chang, 2011). The marked differences in the expression patterns and abundances of mRNAs and IncRNAs imply the distinct biological role that IncRNAs may play in physiological and pathophysiological processes (Esteller, 2011).

IncRNAs have recently been associated with virushost interactions. Josset et al. reported that 5,329 IncRNAs were differentially expressed after influenza A virus and severe acute respiratory syndrome coronavirus (SARS-CoV) infections (Josset et al., 2014). Other studies showed that IncRNAs 7SL and NEAT1 interfere with the HIV-1 virion package and posttranscriptional expression (Wang et al., 2007; Zhang et al., 2013). These data indicate that multiple steps of the virus infection may be regulated by IncRNAs. However, the detailed mechanisms of how IncRNAs are involved during CVA16 infection remain elusive. In this study, we comprehensively characterize the landscape of IncRNAs expressed with or without CVA16 infection in RD cells. Gene Ontology
(GO) (Blake and Harris, 2008) and Kyoto Encyclopedia of Genes and Genomes (KEGG) (Du et al., 2014) analyses were adopted to predict the possible physiological activities and related signal pathways. Thus, we reported a comprehensive catalog of IncRNAs and provided a transcriptome blueprint to identify novel molecular targets and pathways for the treatment of HFMD.

\section{MATERIALS AND METHODS}

\section{Cells and viruses}

RD cells were purchased from the American Type Culture Collection (ATCC) and maintained in minimum essential medium (MEM) supplemented with $10 \%$ fetal bovine serum (FBS) (SV30087; HyClone) or 2\% FBS (maintenance medium). The cells were cultured at $37^{\circ} \mathrm{C}$ in a humidified incubator with $5 \% \mathrm{CO}_{2}$. CVA16 is a laboratory strain that has been completely sequenced and belongs to the B1 genotype (Shi et al., 2015).

\section{RNA extraction and real-time PCR}

The total RNA was extracted using TRIzol reagent (Invitrogen, California, USA) according to the manufacturer's guidelines. Total RNA $(2 \mu \mathrm{g})$ was reverse transcribed into $20 \mu \mathrm{L}$ cDNA using a Reverse Transcription kit (Thermo, Massachusetts, USA) according to the manufacturer's protocol. The cDNA samples were subjected to real-time PCR (Bio-Rad iQ5; Bio-Rad, California, USA) using SYBR green (Gene Copoeia Inc., Maryland, USA) and GAPDH as an internal control. The primers are listed in Table 1. To determine the IncRNAs levels, the following conditions were used: $94{ }^{\circ} \mathrm{C}$ for $5 \mathrm{~min}$, followed by 40 cycles of $94{ }^{\circ} \mathrm{C}$ for $10 \mathrm{~s}, 57-65^{\circ} \mathrm{C}$ for $20 \mathrm{~s}$ and $72{ }^{\circ} \mathrm{C}$ for $30 \mathrm{~s}$. All samples were run in triplicate, and the data analysis was performed using the $2^{-\Delta \Delta \mathrm{Ct}}$ method.

\section{RNA-seq and analysis}

RD cells were infected with CVA16 until cytopathic effects were observed. Total RNA was isolated from each sample by using TRIzol reagent (Invitrogen) according to the manufacturer's protocol. The RNA concentration of each sample was determined by measuring the absorption at 260 and $280 \mathrm{~nm}$ using Nanodrop (Genergy Co., Shanghai, China). High-throughput RNA-Seq of the two mixed samples was performed on Illumina Hiseq2500 with a 101 bp double-end protocol (Genergy Co., Shanghai, China) (Trapnell et al., 2010). Given that the poorquality fractions of the sequencing data were highly distributed in the end and Trim Galore software was used to dynamically remove the poor-quality segments (Wang et al., 2012). Then, FastQC software was adopted for quality control analysis. Subsequently, TopHat was used to map the pre-proposed reads to Homo_sapiens GRCh37 
A

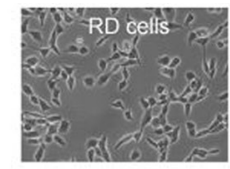

$\Omega$

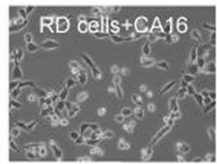

ᄀᄀ

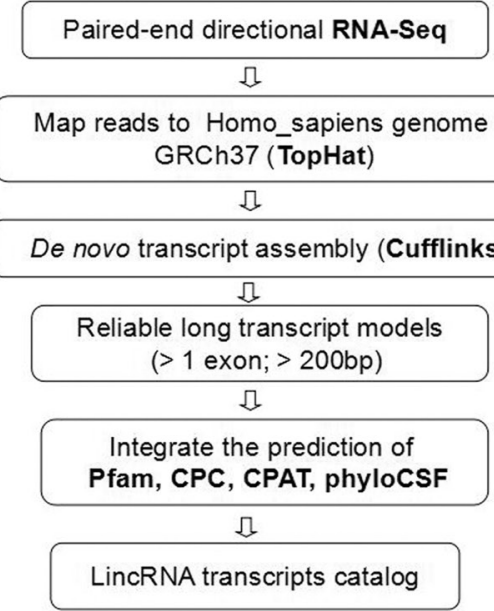

B

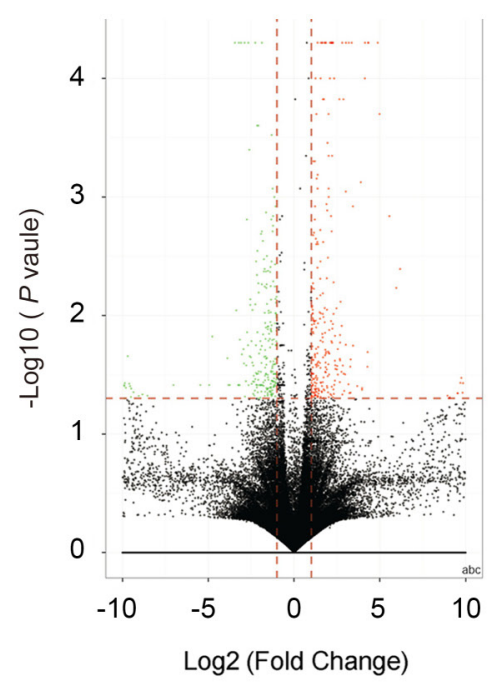

Condition

- abs (log2 (fold change) $)<1, P$ vaule $>0.05$

- $\log 2$ (fold change) $\geq 1, P$ vaule $\leq 0.05$

log2 (fold change) $\geq-1, P$ vaule $\leq 0.05$

\section{C}
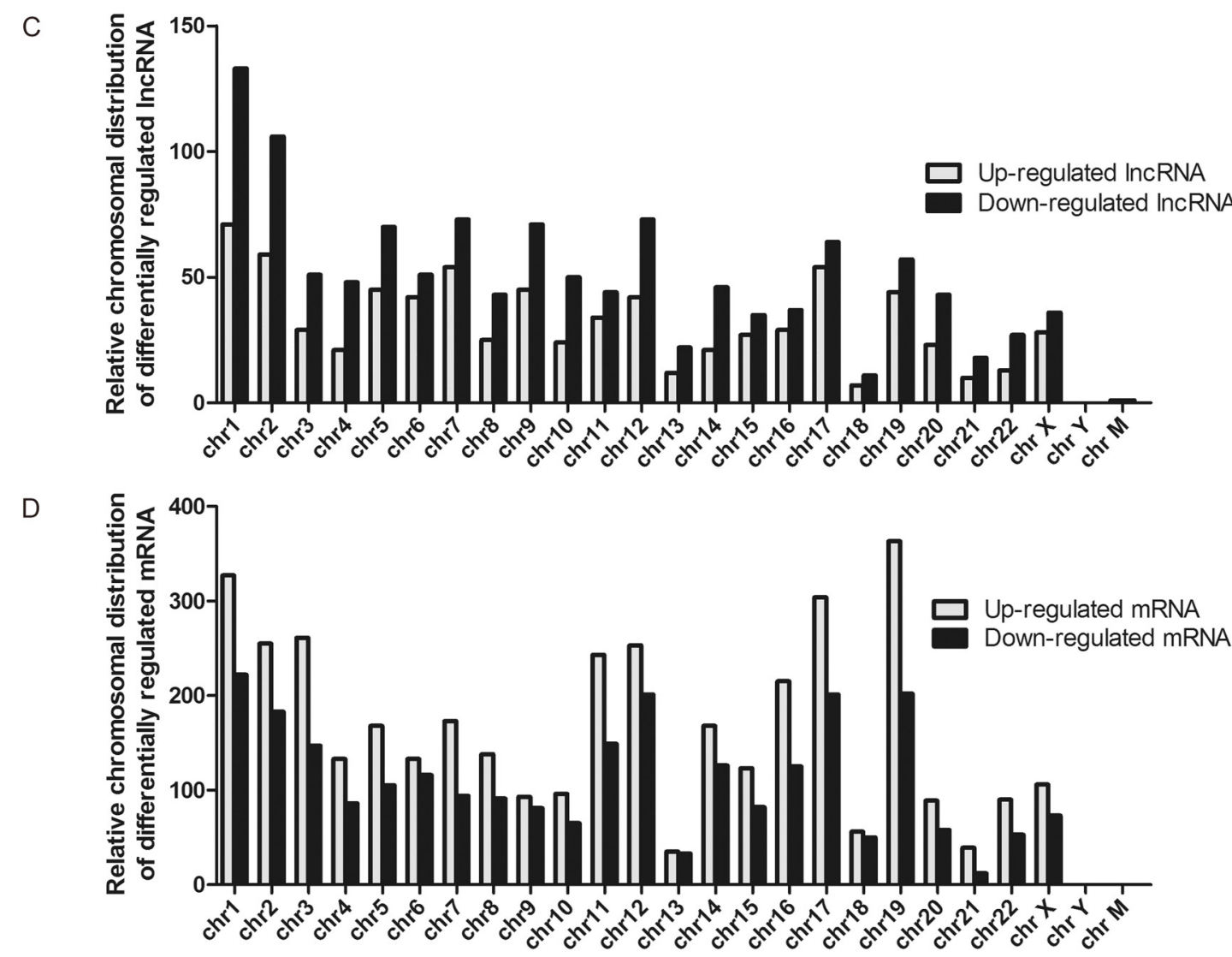

Figure 1. The global discovery of IncRNAs and mRNA expressed in control and CVA16 infected RD cells. (A) Workflow for IncRNAs discovery. The detailed process can be found in the materials and methods. (B) Volcano plots of IncRNAs with significant differential expression. X-axis: log2 fold change; Y-axis: - $\log 10$ (FDR -value); Vertical dotted lines: 2 fold changes in expression level; Horizontal dotted line: the significance cutoff. (C-D) Relative distribution of expressed IncRNAs and mRNA derived from each chromosome. 
and analyze the mapping results to identify splice junctions between the exons (Trapnell et al., 2009). The mapping reads were arranged using Cufflinks to assemble transcripts and estimate their abundance (Borodina et al., 2011). For IncRNAs analyses, the Ensembl, Gencode, NCBI RefGene, UCSC lincRNA, Lncipedia and Noncode databases were chosen as annotation references. For mRNA analyses, we adopted RefSeq and Ensembl databases. Candidate IncRNAs were acquired by satisfying the following criteria: RNA length $\geq 200 \mathrm{nt}, \mathrm{CPC}$ score $\leq$ 0, CPAT probability $\leq 0.364$ and phyloCSF score $\leq-20$. The expression levels of the transcripts were calculated by fragments per kilobase of transcript per million fragments mapped (FPKM) values. Differentially expressed transcripts (DETs) were defined as $P<0.05$ and/or fold change $>2$ times based on their FPKM values between the groups, which were identified using Cuffdiff software (Trapnell et al., 2010).

\section{Secondary structure prediction}

Secondary structure analysis was performed with RNAfold (Vienna package, http://rna.tbi.univie.ac.at/cgibin/RNAfold.cgi). The structure is a minimal free energy structure and base pairing probabilities have been color coded using a scale from 0 (blue) to 1 (red).

\section{GO and KEGG pathway enrichment analyses}

Gene ontology (GO) was adopted to annotate the functions of differentially expressed genes in the GO vocabularies (Blake and Harris, 2008). Briefly, the differentially expressed genes were regarded as candidates from the whole genes and the differentially expressed genes were calculated using a hypergeometric distribution test. The $P$-value was further corrected by Benjamini-Hochberg multiple test to obtain the false discovery rate (FDR) and based on $P$-value and FDR, the enrichment score was expressed in -log 10 ( $P$-value).

Kyoto Encyclopedia of Genes and Genomes (KEGG) pathway analysis was also used to define the functions of the differentially expressed genes in graphical diagrams of biochemical pathways (Du et al., 2014). KEGG pathway analysis was similar to that of GO functional analysis. The significance was calculated by FDR and $P$-value.

\section{Cis and trans analyses}

IncRNAs can regulate the expressions of genes that are located on the same chromosome, and such regulation is called cis regulation (Huang et al., 2012; Kornienko et al., 2013). Several classes of IncRNAs have been reported to regulate their protein-coding host genes in cis manners such as sense intronic, natural antisense transcripts, bidirectional and long intergenic non-coding RNA (Huang et al., 2012; Kornienko et al., 2013). In the present study, we subjected the differentially expressed known IncRNAs to cis analysis by built-in perl code pro- gram.

IncRNAs also can act in a trans manner when they affect genes on other chromosomes (Gong and Maquat, 2011; Huang et al., 2012). Previous studies have shown that IncRNAs can interact with the 3'untranslated region (3'UTR) of its co-expression mRNA, forming complementary hybrids (Gong and Maquat, 2011). Therefore, using the RNAplex and Risearch program (Tafer and Hofacker, 2008; Wenzel et al., 2012), we subjected the differentially expressed known IncRNAs to trans analysis.

\section{RESULTS}

The global discovery of IncRNAs expressed in control and CVA16 infected RD cells

To investigate the roles of host IncRNAs in CVA16 infection, genome-wide RNA-seq was performed using human RD cells infected with or without CVA16. A total of 204 million reads were obtained. The control sample resulted in 95 million reads, and the virus-infected sample resulted in 109 million reads. We mapped RNAseq reads to the human reference genome using TopHat. Transcripts were reconstructed using Cufflinks. HMMer+Pfam, CPC, PhyloCSF and CPAT were used to calculate the coding potential of transcripts. The transcripts that passed HMMer+Pfam, CPC, PhyloCSF and CPAT coding potential filters were further selected as potential IncRNAs. Our stringent strategy is summarized in Figure 1A. Our transcriptome contained a total of 1, 970 IncRNAs and 6, 416 mRNAs with differential expression. Using the criteria of a corrected $\log 2$ (fold_change) $\geq 2$, we identified 760 upregulated and 1, 210 downregulated IncRNAs (Figure 1B, Supplementary Table S1). These IncRNAs were significantly and differentially expressed in CVA16 infected cells compared to the control. Out of the 6, 416 mRNAs, 3, 861 were upregulated and 2, 555 downregulated (Supplementary Table S2). Next, we explored the relative distribution of expressed IncRNAs and mRNAs derived from each chromosome. We found that these reads are ubiquitously distributed in all chromosomes including sex and mitochondria chromosomes (Figure 1C, 1D) (Yang et al., 2014). In addition, the expression levels of IncRNAs were markedly lower than mRNAs, which is consistent with previous reports (Dinger et al., 2008).

\section{General characteristics of dysregulated IncRNAs}

We summarized the general characteristics of dysregulated IncRNAs, such as length distribution, exon number and classification. The majority of the IncRNAs consist of a few exons (less than 5 exons) (Figure 2A). Most IncRNAs are short, with a majority less than $4 \mathrm{~kb}$ in length (Figure 2B).

Based on their genomic locations relative to adjacent 
protein-coding genes, IncRNAs can be divided into several categories: sense, antisense, intergenic, intronic IncRNAs, enhancer IncRNAs or bidirectional IncRNAs (Figure 2C). In the current study, we classified the dysregulated IncRNAs and discovered that $43.64 \%$ were intergenic, $22.31 \%$ were sense, $15.89 \%$ were intronic,

A

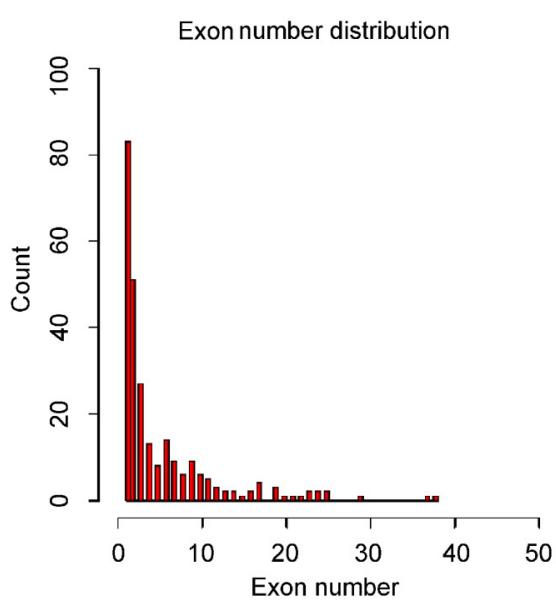

C

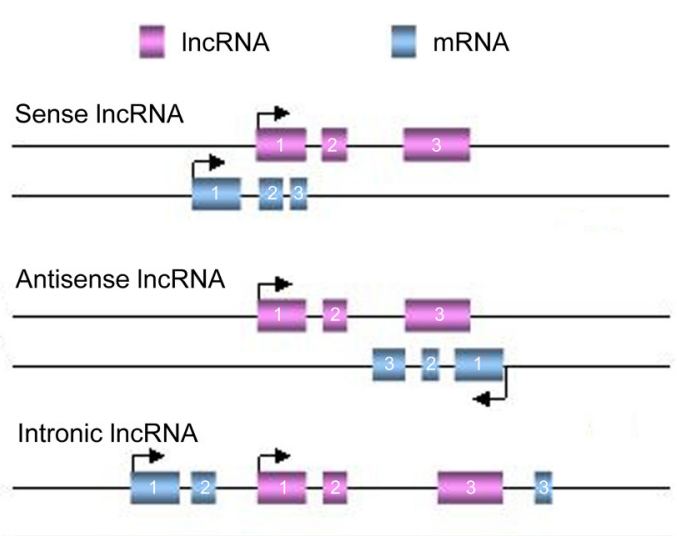

sRNA host IncRNA
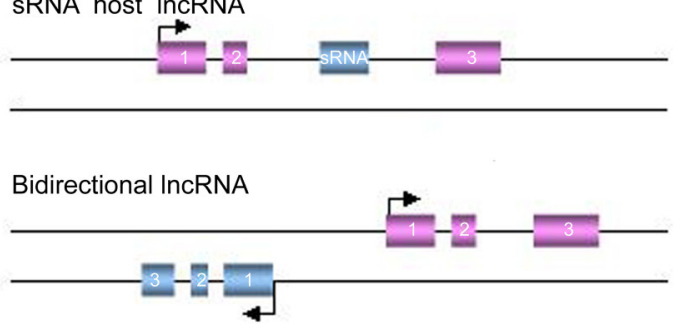

Intergenic IncRNA

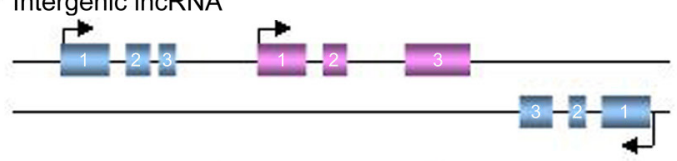

Enhancer IncRNA

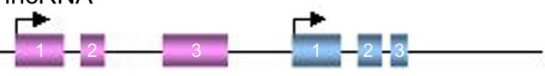

$8.67 \%$ were bidirectional, $5.59 \%$ were antisense, $3.85 \%$ were sRNA host IncRNAs and $0.05 \%$ were enhancer (Figure 2D). Among the successfully annotated IncRNAs, more intergenic transcripts than antisense or intronic transcripts were differentially expressed, which were consistent with previous other investigations (Chen et al.,

B

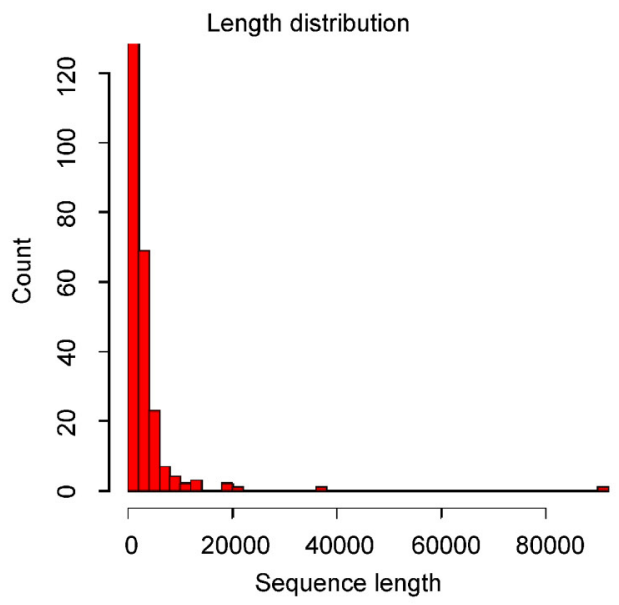

D

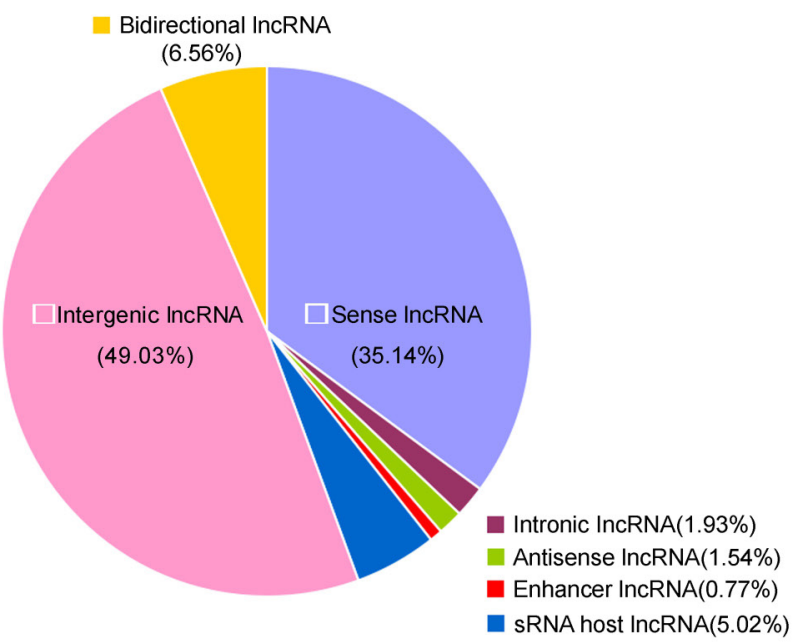

Figure 2. The properties of novel IncRNAs. (A) Number of exons. (B) Size distribution of IncRNAs. (C) Definitions of different classes of IncRNAs based on their genomic region of origin. (D) Annotation of the genomic context of differentially expressed IncRNAs. 
2015; Yu et al., 2015).

\section{Quantitative assays of dysregulated IncRNAs after CVA16 infection}

In this study, to further validate the accuracy of IncRNAs profile determined by RNA-seq, six IncRNAs which are less than $10 \mathrm{~kb}$ were selected as candidates and confirmed by semi-quantitative RT-PCR (Figure 3A, 3B) and quantitative real-time PCR (Figure 3C). As shown in Figure 3C, the real-time PCR results indicate that in the CVA16 infected RD cells the expression levels of NONHSAT102806 and ENST00000307533 increased 2 fold and 4 fold respectively. Meanwhile, the RNA-seq data reveal that the fold change of NONHSAT 102806 is 2.6 and ENST00000307533 is 3.2 upon CVA16 infection (Figure 3D). Next, we compared the real-time PCR results of down-regulated IncRNAs with the RNASeq results and discovered that the changed trends and fold change of real-time PCR results are also consistent with the magnitude of RNA-seq (Figure 3B, 3D). Collectively, these data demonstrated that the realtime PCR results of up/down-regulated IncRNAs are consistent with the levels of differential expression.

\section{KEGG-pathway analysis}

The GO project provided a powerful tool to construct and use ontologies to facilitate the biologically meaningful annotation of genes and their products in a wide variety of organisms (Blake et al., 2008). Genes differentially expressed upon CVA16 infection were analyzed for GO enrichment using the GOseq software package and the top GO terms (based on $P$ value) for cellular component, molecular function, and biologic process for
A

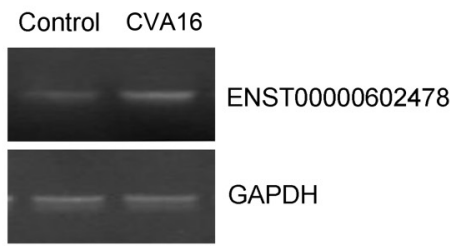

B

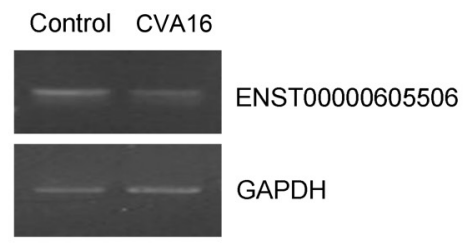

C

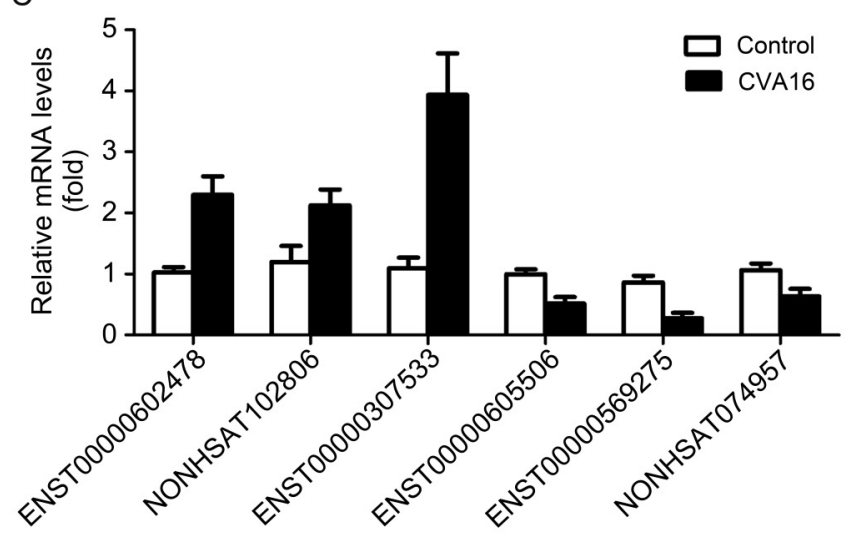

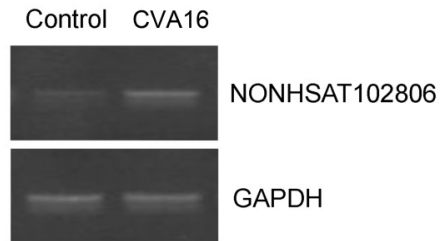
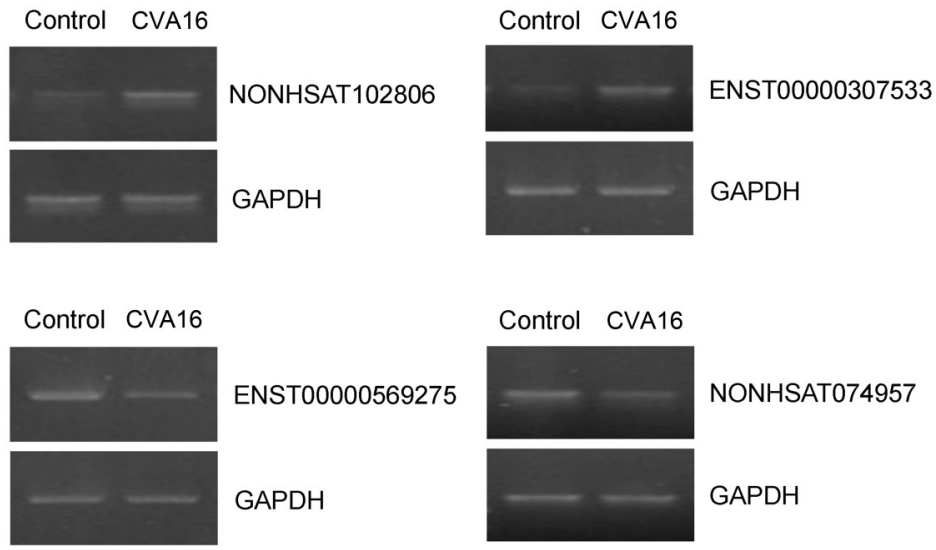

D

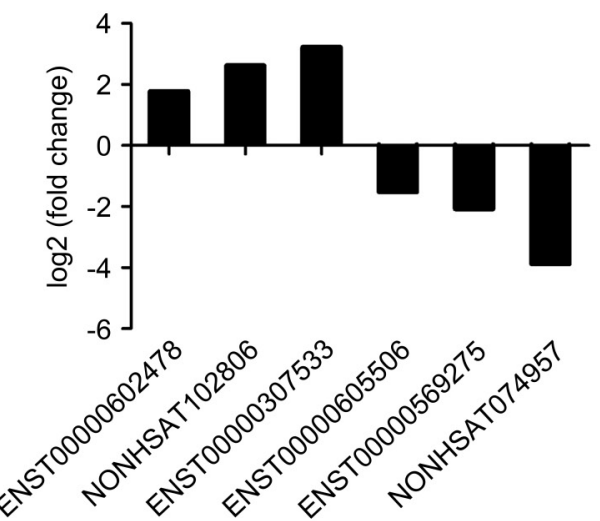

Figure 3. The expression of 6 differentially expressed IncRNAs was validated by semi-quantitative RT-PCR and quantitative real-time PCR. (A-B) Three upregulated IncRNAs and three downregulated IncRNAs, with meaningful elevation or reduction in expression fold demonstrated by RNA-seq assays, were further analyzed and confirmed using semi-quantitative RT-PCR (gel image). (C) Quantitative real-time PCR (column graph) results. (D) RNA-seq results of 6 selected IncRNAs. 
A

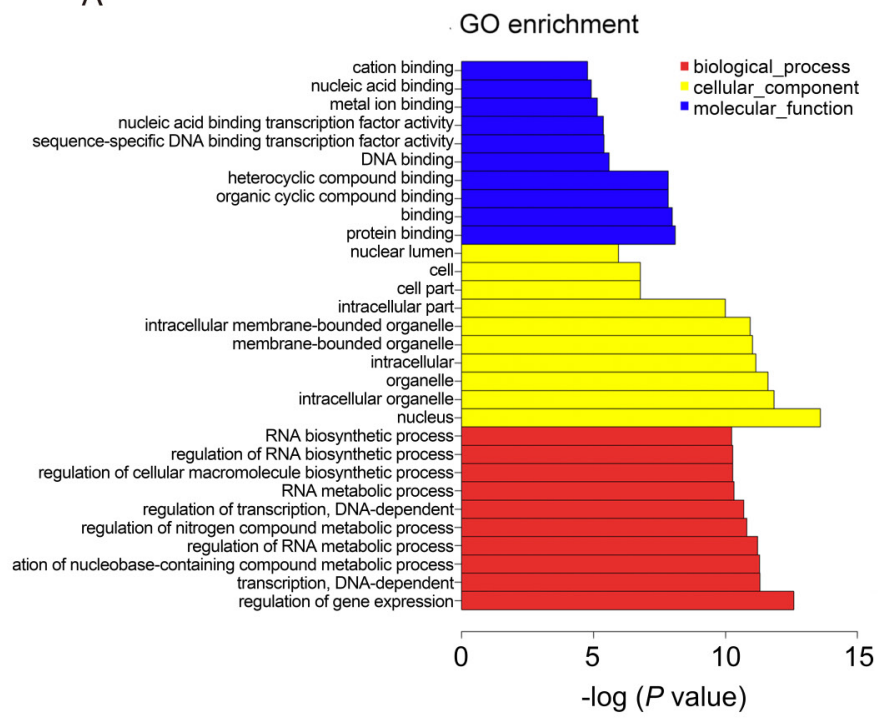

C

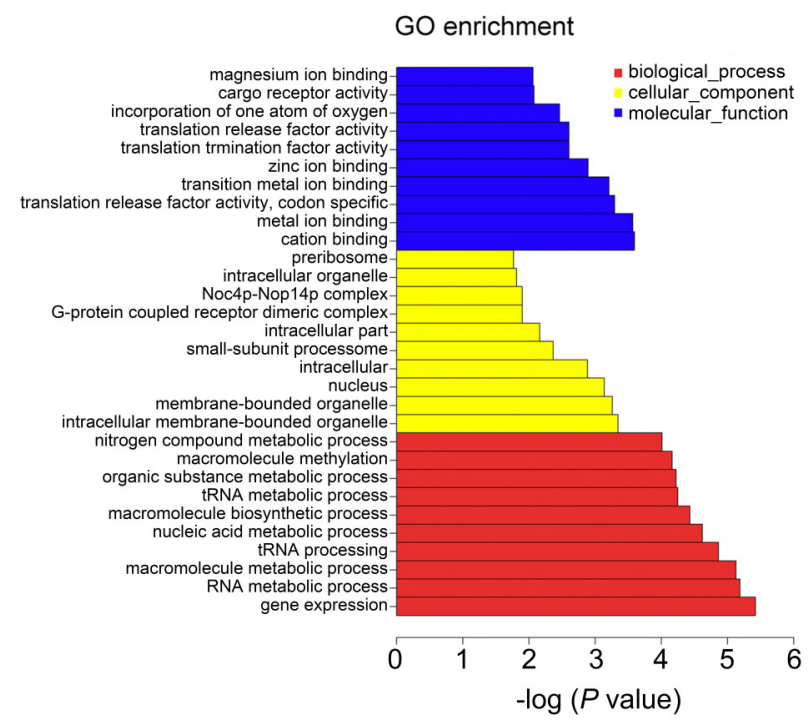

B

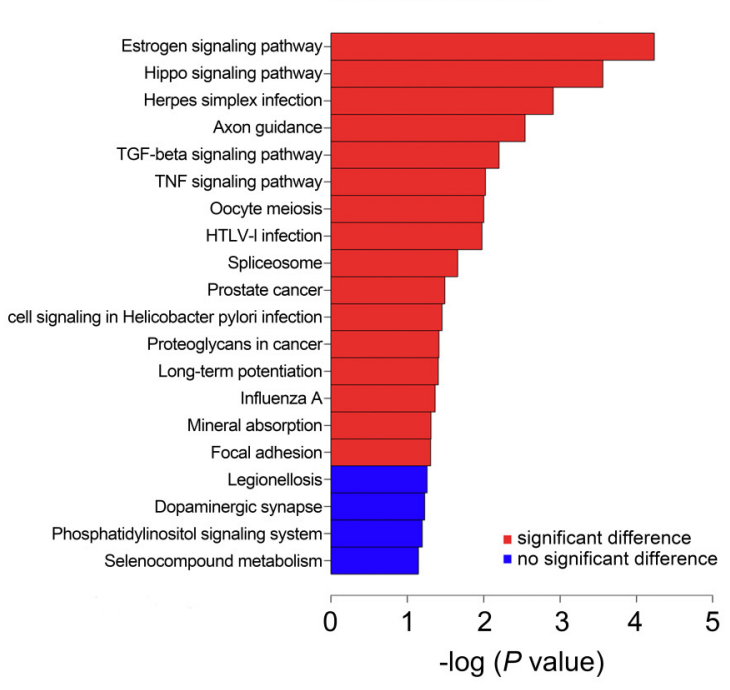

D

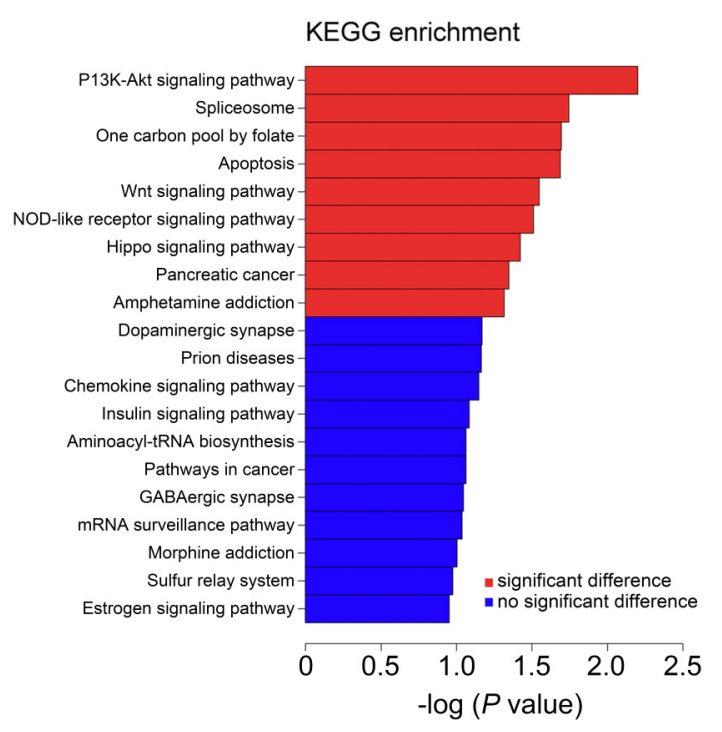

Figure 4. GO and KEGG pathway analysis. (A) The top $10 \mathrm{GO}$ terms of the differentially expressed genes are listed. (B) The pathways that associated with the differentially expressed genes are listed. (C) The top $10 \mathrm{GO}$ terms of the genes that are targeted by differentially expressed IncRNAs. (D) The pathways that were associated with the genes that are targeted by differentially expressed IncRNAs are listed.

differentially expressed genes are shown in Figure 4A.

For cellular component terms, genes differentially expressed are enriched for components of the nucleus, membrane-bounded organelle, intracellular membranebounded organelle and intracellular part (Figure 4A).

For molecular function terms, differentially expressed genes show enrichment for transcripts that encode nucleic acid binding transcription factor activity, sequencespecific DNA binding transcription factor activity. Transcripts encoding binding activities, include protein binding, DNA binding, metal ion binding, nucleic acid binding, heterocyclic compound binding and organic cyclic compound binding are also abundantly expressed in CVA16 infected RD cell fraction (Figure 4A).

Biologic process terms enriched in differential expressed genes include regulation of gene expression, transcription, regulation of RNA metabolic process and regulation of transcription (Figure 4A). This is consistent with the fact that RD cells undergo changes of gene expression regulation after CVA16 infection (Figure 4A).

Next, these genes were subjected to KEGG database analysis to predict the biological pathways associated with the differentially expressed genes (Du et al., 2014). 
The top 16 significant pathways associated with the differentially expressed genes were shown (Figure 4B). The results of KEGG analysis showed that they were mainly enriched for TGF-beta signaling pathway, TNF signaling pathway and virus infection related signaling pathway (influenza A, HTLV-1 infection).

Target genes of differentially expressed IncRNAs were predicted and these target genes also were subjected to GO annotation and KEGG pathway analysis (Figure 4C, 4D). The results of GO annotation demonstrated the main functions of these target genes were associated with magnesium ion binding, cargo receptor activity, nitrogen compound metabolic processes, molecular methylation and organic substance metabolic processes (Figure 4C). While the results of KEGG analysis showed they were mainly related to the following signal pathways: (1) the PI3K-Akt signaling pathway, (2) the splicesome, (3) apoptosis, (4) the Wnt signaling pathway, (5) the NOD-like receptor signaling pathway, (6) the Hippo signaling pathway and (7) pancreatic cancer (Figure 4D).

\section{Cis-acting and trans-acting regulatory network}

IncRNAs can work in cis or trans manner when they affect genes on the same or different chromosomes (Gong and Maquat, 2011; Huang et al., 2012; Kornienko et al., 2013). Identification of the genes associated with differentially expressed IncRNAs via cis-or trans-regulation might provide insight into the potential functions of IncRNAs. In the present study, we subjected the differentially expressed IncRNAs to $c i s$ analysis, and we found that hundreds of the differentially expressed known IncRNAs could act in a cis manner. As shown in Supplementary Figure S1A, we know that lnc-TENC1-1:1 was associated with eukaryotic translation initiation factor $4 \mathrm{~B}$ (eIF4B) which is an important protein involved in the initiation phase of eukaryotic translation (Supplementary Table S3). Meanwhile, mitogen-activated protein kinase 9 (MAPK9) are predicted to be associated with UCSC_TCONS_00010210 (Supplementary Table S3).

In addition, we also subjected the differentially expressed IncRNAs to trans -analysis and found that transacting IncRNAs may not be as prevalent as cis-acting IncRNAs. In addition, we further detected the networks formed by the trans-acting IncRNAs and their associated genes and found that one IncRNAs can have one or more associated genes. As shown in Supplementary Figure S1B, NONHSAG051892 not only was predicted to be associated with Ribonuclease P protein subunit $\mathrm{p} 38$ but also Galactokinase in trans manner. More detailed information can be found in Supplementary Table S3 and Supplementary Table S4.

\section{Further analysis about selected IncRNAs}

The function of an IncRNAs cannot be inferred from the sequence or primary structure alone (Mercer et al., 2009; Wang and Chang, 2011; Rybarczyk et al., 2015). Indeed, there is already strong evidence that evolutionarily conserved RNA secondary structures are a robust indicator of molecular function. Based on the predicted secondary structure of IncRNAs NRAV, Jing Ouyang et al. conducted a series of related experiments and demonstrated that a small arm of NRAV (nt 618-872) was non-essential for its role in controlling IAV replication (Ouyang et al., 2014). In the present study, we also predicted the second structure of six IncRNAs and we discovered various pseudoknots or stem loops in these IncRNAs (Supplementary Figure S2). It is attempting to estimate that the flexible and complex structures of the RNA may be related to the diversity of their functions and whether these stem loops play vital roles need to be validated further.

To characterize the distinctive chromatin structures, histone modifications and transcription factor binding signatures, we selected ENST00000602478 as an example for further research. We found that ENST00000 602478 is a natural antisense IncRNAs that is mapped on chromosome 22 (Figure 5A). Two protein coding genes on the opposite strand transcribe in the opposite direction (Figure 5A). One is Cytochrome-b5 reductase (methemoglobin reductase), an NADH-dependent enzyme that converts methemoglobin to hemoglobin. The other protein is the polymerase delta-interacting protein 3 , a protein that interacts with the DNA polymerase delta p50 subunit and is also a specific target of S6 kinase 1 (regulates cell growth). Exon of ENST00000602478 shows high conservation across mammalian species (Figure 5B). Further, there is a prominent histone H3-lysine27 acetylation (H3K27ac) marker near exon of ENST00000602478, suggesting the presence of an active promoter (Figure 5B). Further experiments, such as chromatin immunoprecipitation sequencing (ChIP-seq) or multisite ChIP-quantitative polymerase chain reaction (qPCR), are necessary to verify the histone modifications near the IncRNAs loci. Another track DNase I hypersensitivity peak clusters also are enriched in transcription start sites (TSS) of ENST00000602478 (Figure 5B), leading to increased chromatin accessibility. In addition, in the IncRNAs promoter region, we report various binding motifs $(+1000$ to $0 \mathrm{nt}$ relative to transcription start sites (TSS)) for transcription factors which may regulate IncRNAs expression (Figure 5C). And the critical roles for these transcription factors need to be confirmed by luciferase assays. Collectively, these features indicated that the expression of IncRNAs can be regulated by various mechanisms.

\section{DISCUSSION}

The present study utilized next-generation sequencing to 
A

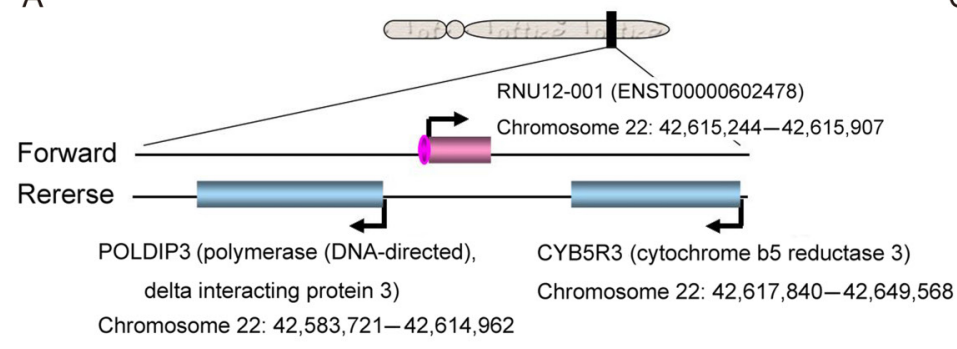

C

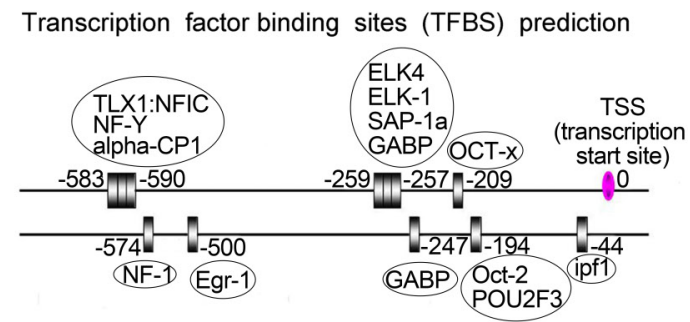

B

UCSC genome browser track of RNU12 (ENST00000602478)

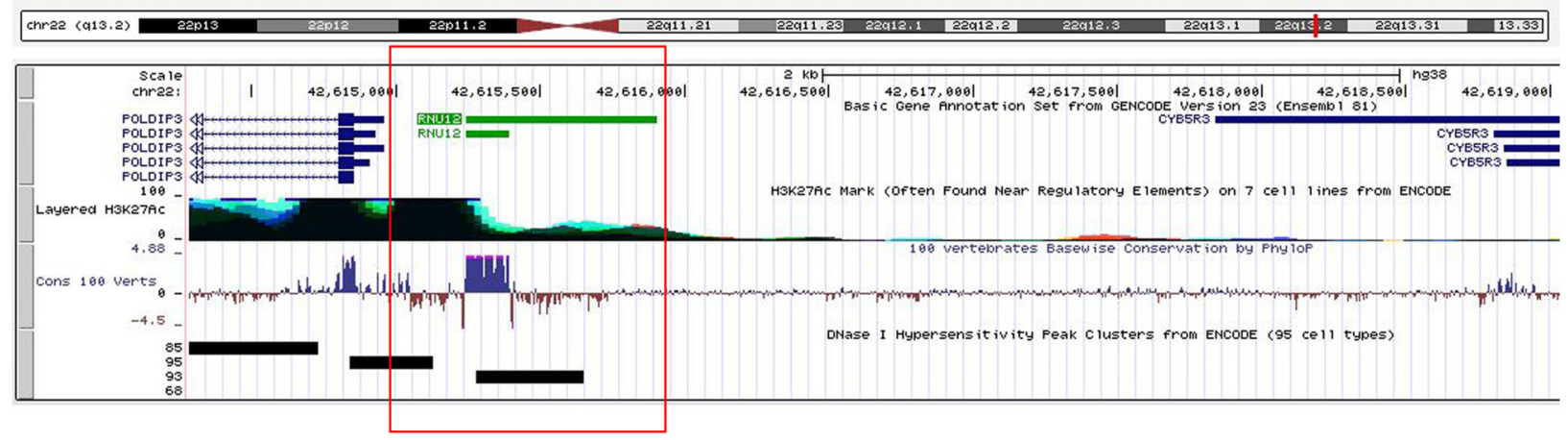

Figure 5. A bioinformatics analysis of ENST00000602478. (A) The position of ENST00000602478 and neighboring genes in chromosome 22. (B) Several integrated regulation tracks spanning the ENST00000602478, including conservation, histone markings and DNase I hypersensitivity, are displayed. (C) Transcription factor binding sites (TFBS) that are located in the IncRNAs promoter region (-1000 to $0 \mathrm{nt}$ relative to TSS) were predicted by MotifMap software. The transcription factors (TF) were in the oval boxes and the numbers indicated the binding sites.

provide a quantitative and comprehensive analysis of the coding and non-coding transcriptome of mock-infected and CVA16 infected RD cells. These analyses revealed significant differences in the patterns of mRNA and IncRNAs expression. A total of 1, 970 IncRNAs and 6, 416 mRNAs were identified as significantly and differentially expressed between control and CVA16 infected cells. GO analysis and KEGG analysis were conducted to investigate the potential functions of these IncRNAs. Our study is the first to use comprehensive deep-sequencing technology to clearly demonstrate that IncRNAs are involved in the host response to CVA16 infection.

A number of recent studies have suggested that noncoding RNAs (ncRNAs) function in pathogen-host interactions (Peng et al., 2010; Winterling et al., 2014). Using next-generation sequencing, Peng et al. reported the differential expression of approximately 500 annotated and 1,000 non-annotated genomic regions during SARS$\mathrm{CoV}$ infection across four founder mouse strains (Peng et al., 2010). Using two commercially available microarray systems, Winterling et al. identified the differential expression of 42 ncRNAs during influenza A virus (IAV) infection in human lung epithelial cells and IncRNAs VIN can facilitate influenza A virus (IAV) propagation
(Winterling et al., 2014). Despite these progresses, the specific functions of these IncRNAs remain incompletely characterized and these virus infection models provide a unique platform for studying the biology and regulation of IncRNAs.

Unlike mRNAs, IncRNAs possess some unique properties. In this study, we revealed that the expression levels of most IncRNAs are lower than mRNA. We also noted that more intergenic IncRNAs than antisense and intronic IncRNAs were differentially expressed. This result may be an intrinsic property of organism system associated with the respective functions of non-coding genes and protein-coding genes. Another interesting finding was that the numbers of down-regulated IncRNAs are unexceptionally more than that of up-regulated IncRNAs in each chromosome, and the result of mRNA is quite the opposite. Although this result is consistent with previous other investigations ( $\mathrm{Bu}$ et al., 2012; Zhu et al., 2015), Yu et al. reported that there were more down-regulated IncRNAs than up-regulated IncRNAs in the glucocorticoids-treated bone microvascular endothelial cells and conversely, differentially upregulated mRNAs were more common than significantly down-regulated mRNAs (Yu et al., 2015). The precise 
regulatory mechanism remains unclear and further studies are needed to illuminate the exact mechanisms. Taken together, these results demonstrate that our IncRNAs share similar features with those described in previous studies, in terms of structural, expression, and conservation properties (Alvarez-Dominguez et al., 2014; Yang et al., 2014; Chen et al., 2015).

Although the development of high throughput deep sequencing technology provided the possibility of a nearly complete view of IncRNAs profiles, the identification of IncRNAs function still remains challenging (Wang and Chang, 2011; Djebali et al., 2012). The GO project and KEGG Pathway analysis are widely used to illustrate the differentially expressed genes in terms of functions and pathways. In this study, we detected that the dysregulated genes were enriched in RNA biologic processes including RNA biosynthetic processes, RNA metabolic processes and the regulation of RNA upon CVA16 infection (Figure 4A). The coding gene targeted by dysregulated IncRNAs shown enrichment in the PI3K-Akt signaling pathway, splicesome, apoptosis, Wnt signaling pathway and the NOD-like receptor signaling pathway (Figure 4D). The PI3K-Akt signaling pathway regulates many cellular processes including development, cell proliferation, differentiation, and apoptosis. The NOD-like receptor signaling pathway plays key roles in the regulation of the innate immune response by cooperating with Toll-like receptors and regulating inflammation. Therefore, the GO project and KEGG Pathway analysis provide guidance for further efficient identification of potential functions and regulatory mechanism of IncRNAs. And further experimental verifications are needed to verify the hypothesis.

IncRNAs can regulate gene expression either in a cis (on neighboring genes) or in a trans (on distantly located genes) manner (Mercer et al., 2009; Wang and Chang, 2011; Guil and Esteller, 2012; Lee, 2012). Although an increasing number of IncRNAs are reported, only a small fraction of them have functional annotations. Most IncRNAs in a given cellular content remain enigmas. In the current study, we analyzed IncRNAsmRNA genomic proximity information and constructed a co-expression network to explore the potential cis- and trans-regulatory roles of IncRNAs on coding mRNAs. We discovered that numerous IncRNAs interacted with associated protein-coding genes in cis or trans manners, which suggests that these IncRNAs might be biologically meaningful. Notably, these IncRNAs were found to form a "many-to-many" network with their associated genes, which reflects the complexity of the mechanisms of the regulation of CVA16-regulated IncRNAs. The IncRNAs and mRNA co-expression networks provide concrete targets for further function research of IncRNAs. Further experiments are needed to investigate the pre- cise natures of these IncRNAs.

These studies utilized next-generation sequencing technologies for comprehensive mRNA and IncRNAs expression profiling in control and CVA16 infected RD cells. These experiments revealed a distinct relative abundance, expression pattern and genomic origin of mRNA and IncRNAs in human RD cells, highlighting the different biological roles of the individual RNA classes. Further study of the nature and function of these dysregulated IncRNAs is necessary to determine the mechanisms of HFMD. All in all we identified a panel of IncRNAs derived from CVA16 infected RD cells, which may provide new targets for the diagnosis, treatment and prevention of HFMD.

\section{ACKNOWLEDGMENTS}

This work was supported by the National Natural Sciences Foundation of China (No. 81171577, 81371790, 81371422 and 81171127), Major AIDS and Viral Hepatitis and Other Major Infectious Disease Prevention and Control project of China (2014ZX10001003), the Fundamental Research Funds for the Central Universities of China and the Translational Medical Research Fund of Wuhan University School of Medicine.

\section{COMPLIANCE WITH ETHICS GUIDELINES}

The authors declare that they have no conflict of interest. This article does not contain any studies with human or animal subjects performed by any of the authors.

\section{AUTHOR CONTRIBUTIONS}

WHL and YYS designed the experiments, analyzed the data and wrote the paper. YYS and HLT performed the majority of the experiments. XC, YYZ, LJC and ZCL offered some experimental materials. JQS, SH, JY, BWP, XHH and WHL supervised this study and reviewed and edited the paper.

Supplementary figures/tables are available on the website of Virologica Sinica: www.virosin.org; link. springer. com/journal/12250.

\section{REFERENCES}

Alvarez-Dominguez J R, Hu W, Yuan B, Shi J, Park S S, Gromatzky A A, van Oudenaarden A, Lodish H F. 2014. Global discovery of erythroid long noncoding RNAs reveals novel regulators of red cell maturation. Blood, 123: 570-581.

Bertone P, Stolc V, Royce T E, Rozowsky J S, Urban A E, Zhu X, Rinn J L, Tongprasit W, Samanta M, Weissman S, et al. 2004. Global identification of human transcribed sequences with genome tiling arrays. Science, 306: 2242-2246. 
Blake JA, Harris MA. 2008. The Gene Ontology (GO) project: structured vocabularies for molecular biology and their application to genome and expression analysis. Curr Protoc Bioinformatics. doi: 10.1002/0471250953.bi0702s23.

Borodina T, Adjaye J, Sultan M. 2011. A strand-specific library preparation protocol for RNA sequencing. Methods Enzymol, 500: 79-98.

Bu Q, Hu Z, Chen F, Zhu R, Deng Y, Shao X, Li Y, Zhao J, Li H, Zhang B, et al. 2012. Transcriptome analysis of long non-coding RNAs of the nucleus accumbens in cocaine-conditioned mice. J Neurochem, 123: 790-799.

Chen X, Tan X, Li J, Jin Y, Gong L, Hong M, Shi Y, Zhu S, Zhang B, Zhang S, et al. 2013. Molecular epidemiology of coxsackievirus A16: intratype and prevalent intertype recombination identified. PLoS One, 8: e82861.

Chen Z, Luo Y, Yang W, Ding L, Wang J, Tu J, Geng B, Cui Q,Yang J. 2015. Comparison Analysis of Dysregulated IncRNAs Profile in Mouse Plasma and Liver after Hepatic Ischemia/Reperfusion Injury. PLoS One, 10: e0133462.

Dinger ME, Amaral PP, Mercer TR, Pang KC, Bruce SJ, Gardiner BB, Askarian-Amiri ME, Ru K, Solda G, Simons C, et al. 2008. Long noncoding RNAs in mouse embryonic stem cell pluripotency and differentiation. Genome Res, 18: 1433-1445.

Djebali S, Davis C A, Merkel A, Dobin A, Lassmann T, Mortazavi A, Tanzer A, Lagarde J, Lin W, Schlesinger F, et al. 2012. Landscape of transcription in human cells. Nature, 489: 101-108.

Du J, Yuan Z, Ma Z, Song J, Xie X, Chen Y. 2014. KEGG-PATH: Kyoto encyclopedia of genes and genomes-based pathway analysis using a path analysis model. Mol Biosyst, 10: 2441-2447.

Esteller M. 2011. Non-coding RNAs in human disease. Nat Rev Genet, 12: 861-874.

Fatica A, Bozzoni I. 2014. Long non-coding RNAs: new players in cell differentiation and development. Nat Rev Genet, 15: 7-21.

Gomez JA, Wapinski OL, Yang YW, Bureau JF, Gopinath S, Monack DM, Chang HY, Brahic M, Kirkegaard K. 2013. The NeST long ncRNA controls microbial susceptibility and epigenetic activation of the interferon-gamma locus. Cell, 152: 743754.

Gong C,Maquat LE. 2011. IncRNAs transactivate STAU1-mediated mRNA decay by duplexing with 3' UTRs via Alu elements. Nature, 470: 284-288.

Guil S, Esteller M. 2012. Cis-acting noncoding RNAs: friends and foes. Nat Struct Mol Biol, 19: 1068-1075.

Huang Y, Liu N, Wang JP, Wang YQ, Yu XL, Wang ZB, Cheng $\mathrm{XC}$, Zou Q. 2012. Regulatory long non-coding RNA and its functions. J Physiol Biochem, 68: 611-618.

Josset L, Tchitchek N, Gralinski L E, Ferris M T, Eisfeld A J, Green RR, Thomas MJ, Tisoncik-Go J, Schroth GP, Kawaoka $\mathrm{Y}$, et al. 2014. Annotation of long non-coding RNAs expressed in collaborative cross founder mice in response to respiratory virus infection reveals a new class of interferon-stimulated transcripts. RNA Biol, 11: 875-890.

Kapranov P, Cheng J, Dike S, Nix DA, Duttagupta R, Willingham AT, Stadler PF, Hertel J, Hackermuller J, Hofacker IL, et al.2007. RNA maps reveal new RNA classes and a possible function for pervasive transcription. Science, 316: 1484-1488.

Kornienko AE, Guenzl PM, Barlow DP, Pauler FM. 2013. Gene regulation by the act of long non-coding RNA transcription. BMC Biol, 11: 59.

Lee JT. 2012. Epigenetic regulation by long noncoding RNAs. Science, 338: 1435-1439.

Mao Q, Wang Y, Yao X, Bian L, Wu X, Xu M, Liang Z. 2014. Coxsackievirus A16: epidemiology, diagnosis, and vaccine. Hum Vaccin Immunother, 10: 360-367.

Mattick JS. 2011. The central role of RNA in human development and cognition. FEBS Lett, 585: 1600-1616.

Mattick JS, Makunin IV. 2006. Non-coding RNA. Hum Mol Genet, 15. doi: 10.1093/hmg/dd1046.

Mercer TR, Dinger ME, Mattick JS. 2009. Long non-coding
RNAs: insights into functions. Nat Rev Genet, 10: 155-159.

Ouyang J, Zhu X, Chen Y, Wei H, Chen Q, Chi X, Qi B, Zhang L, Zhao Y, Gao GF, et al. 2014. NRAV, a long noncoding RNA, modulates antiviral responses through suppression of interferonstimulated gene tran-scription. Cell Host Microbe, 16:616-626.

Peng X, Gralinski L, Armour CD, Ferris MT, Thomas MJ, Proll S, Bradel-Tretheway BG, Korth MJ, Castle JC, Biery MC, et al. 2010. Unique signatures of long noncoding RNA expression in response to virus infection and altered innate immune signaling. MBio, 1. doi: 10.1128/mBio.00206-10.

Ren J, Wang X, Zhu L, Hu Z, Gao Q, Yang P, Li X, Wang J, Shen X, Fry EE, et al. 2015. Structures of coxsackievirus A16 capsids with native antigenicity, implications for particle expansion, receptor binding and immunogenicity. J Virol, 89:10500-10511.

Rybarczyk A, Szostak N, Antczak M, Zok T, Popenda M, Adamiak R, Blazewicz J, Szachniuk M. 2015. New in silico approach to assessing RNA secondary structures with non-canonical base pairs. BMC Bioinformatics, 16: 276

Shi Y, He X, Zhu G, Tu H, Liu Z, Li W, Han S, Yin J, Peng B, Liu W. 2015. Coxsackievirus A16 elicits incomplete autophagy involving the mTOR and ERK pathways. PLoS One, 10: e0122109.

Sun T, Liu Y, Zhang Y, Zhou L. 2014. Molecular phylogeny of coxsackievirus A16. J Clin Microbiol, 52: 3829-3830.

Tafer H, Hofacker IL. 2008. RNAplex: a fast tool for RNA-RNA interaction search. Bioinformatics, 24: 2657-2663.

Trapnell C, Pachter L, Salzberg S L. 2009. TopHat: discovering splice junctions with RNA-Seq. Bioinformatics, 25: 1105-1111.

Trapnell C, Williams BA, Pertea G, Mortazavi A, Kwan G, van Baren MJ, Salzberg SL, Wold BJ, Pachter L. 2010. Transcript assembly and quantification by RNA-Seq reveals unannotated transcripts and isoform switching during cell differentiation. Nat Biotechnol, 28: 511-515.

Wang KC, Chang HY. 2011. Molecular mechanisms of long noncoding RNAs. Mol Cell, 43: 904-914.

Wang L, Wang S, Li W. 2012. RSeQC: quality control of RNAseq experiments. Bioinformatics, 28: 2184-2185.

Wang T, Tian C, Zhang W, Luo K, Sarkis PT, Yu L, Liu B, Yu Y, Yu XF. 2007. 7SL RNA mediates virion packaging of the antiviral cytidine deaminase APOBEC3G. J Virol, 81: 13112-13124.

Wei W, Guo H, Li J, Ren S, Wei Z, Bao W, Hu X, Zhao K, Zhang W, Zhou Y, et al. 2014. Circulating HFMD-associated coxsackievirus A16 is genetically and phenotypically distinct from the prototype CV-A16. PLoS One, 9: e94746.

Wenzel A, Akbasli E, Gorodkin J. 2012. RIsearch: fast RNA-RNA interaction search using a simplified nearest-neighbor energy model. Bioinformatics, 28: 2738-2746.

Winterling C, Koch M, Koeppel M, Garcia-Alcalde F, Karlas A, Meyer TF. 2014. Evidence for a crucial role of a host non-coding RNA in influenza A virus replication. RNA Biol, 11: 66-75. Yang KC, Yamada KA, Patel AY, Topkara VK, George I, Cheema FH, Ewald GA, Mann DL, Nerbonne JM. 2014. Deep RNA sequencing reveals dynamic regulation of myocardial noncoding RNAs in failing human heart and remodeling with mechanical circulatory support. Circulation, 129: 1009-1021.

Yu QS, Guo WS, Cheng LM, Lu YF, Shen JY, Li P. 2015. Glucocorticoids Significantly Influence the Transcriptome of Bone Microvascular Endothelial Cells of Human Femoral Head. Chin Med J (Engl), 128: 1956-1963.

Zhang Q, Chen CY, Yedavalli VS, Jeang KT. 2013. NEAT1 long noncoding RNA and paraspeckle bodies modulate HIV-1 posttranscriptional expression. MBio, 4: e00596-00512.

Zhu G, Zheng Y, Zhang L, Shi Y, Li W, Liu Z, Peng B, Yin J, Liu W, He X. 2013. Coxsackievirus A16 infection triggers apoptosis in RD cells by inducing ER stress. Biochem Biophys Res Commun, 441: 856-861.

Zhu L, Zhu J, Liu Y, Chen Y, Li Y, Huang L, Chen S, Li T, Dang Y, Chen T. 2015. Methamphetamine induces alterations in the long non-coding RNAs expression profile in the nucleus accumbens of the mouse. BMC Neurosci, 16: 18. 